\title{
Molecular-Dynamics Simulation for the Characterization of Liquid Chromatographic Stationary Phase: Effect of Temperature
}

\author{
Kazuhiro BAN, ${ }^{\dagger}$ Yoshihiro SAITO, and Kiyokatsu JinNo \\ Toyohashi University of Technology, School of Materials Science, Toyohashi, Aichi 441-8580, Japan
}

\begin{abstract}
The influence of temperature on the surface structure of the octadecylsilica (ODS) bonded phase was investigated with a molecular dynamics (MD) simulation. The MD simulation was applied to a molecular model consisting of three parts: amorphous silica base, dimethyloctadecylsilyl ligands and $n$-hexane as a mobile phase solvent. More detailed information on the effect of temperature was obtained at the low temperature region than that reported in our previous study. The motion of ODS ligands could be estimated by the mean square displacement (MSD) of the terminal carbon atoms of ODS ligands. The gauche fraction in the ODS ligand conformation can also be estimated to obtain the ligand conformation for each simulation condition in detail. It can be seen that an elevated temperature induced the more bent ligand conformation. The trend has a good agreement to that of the results experimentally observed by using various spectroscopic techniques such as nuclear magnetic resonance (NMR), Fourier-transform infrared spectroscopy (FT-IR), and Raman spectroscopy.
\end{abstract}

(Received December 15, 2004; Accepted January 26, 2005)

\section{Introduction}

Liquid chromatography (LC) is one of the most powerful methods currently used for the separation of a wide range of mixtures in various samples. Despite the widespread use of LC in both analytical and preparative applications, the separation mechanism has not been completely clarified yet. Therefore, the method development and optimization are still often based on a large number of empirical "trial and error" approaches. It is well known that the retention in LC was generated by the partition of the analyte between the mobile phase and the stationary phase. Especially, the intermolecular interaction between the analyte and the stationary phase ligand plays a very important role during the retention process. Therefore, the characterization of the stationary phase is necessary to interpret the retention mechanism in $\mathrm{LC}$.

As a stationary phase in LC, octadecylsilica(ODS)-bonded phase has been most widely used, mainly because of its high separation ability. It is well known that the separation ability can be significantly different depending on the synthetic conditions of the bonded phase. A number of reports have been published for the relationship between the synthetic conditions and separation ability of the ODS bonded phase. These studies used techniques such as nuclear magnetic resonance (NMR) spectroscopy, ${ }^{1-8}$ Fourier-transform infrared spectroscopy (FTIR), ${ }^{5,9}$ Raman spectroscopy, ${ }^{10-17}$ contact angle measurements, ${ }^{18}$ thermal analysis ${ }^{5,19,20}$ and actual chromatographic researches. ${ }^{20-26}$ These results suggested that the separation ability depends on the variation of the surface structure of the stationary phase

$\dagger$ To whom correspondence should be addressed.

E-mail: ban@ chrom.tutms.tut.ac.jp including the density and conformation of the ODS ligands.

It is also known that the column temperature in the LC separation affects the retention and selectivity significantly. ${ }^{27-32}$ Sander et al. have investigated the temperature dependence of selectivity in LC using several types of ODS-bonded phases. ${ }^{27}$ In their report, the selectivity of the bonded phase varied continuously with temperature, and it is not specific to a particular phase type. In our previous investigations, we reported the separation of polycyclic aromatic hydrocarbons (PAHs) and fullerenes in LC using various alkyl-bonded phases and non-aqueous solvents as a mobile phase, $, 200,29-32$ and found that the difference of the ligand interval changes the selectivity especially at low temperature. The temperature dependency on the ligand conformation has also been well researched by using solid state NMR, ${ }^{5,7}$ FTIR, ${ }^{5,9}$ Raman spectroscopy, ${ }^{12-15}$ and thermal analysis. ${ }^{5,19,20}$

Molecular dynamics (MD) simulation is a powerful method to estimate the time series structural changes of a multi-molecular system, and is widely used in biochemistry to characterize the higher structures of biomolecules. The advances of computer technologies have made it possible to use this technique on a personal computer and also to handle easily the calculations for a huge molecular system such as the stationary phase in LC. Some articles and reviews have been reported for the characterization of the stationary phase ligand conformation by using the MD simulation. ${ }^{33-42}$ Klatte and Beck applied the MD simulation to the molecular models, which consisted of the tethered and randomly placed $n$-alkane monolayers. They investigated the influence of temperature, alkyl chain length and the density of alkane molecules on some physical properties such as alkyl chain conformation and phase transition. ${ }^{33,34}$

In our previous study, we have applied the MD simulation on some molecular models that consisted of ODS-bonded phase 
and mobile phase molecules to investigate the influence of mobile phase composition and temperature on the conformation of the octadecylsilyl group. ${ }^{40,42}$ It was clearly shown that the MD simulation is useful to discuss the influence of the mobile phase composition and temperature on the surface structures. In this study, we have applied the MD simulation at subambient temperature on a molecular model containing the ODS bonded phase and mobile phase solvents, and we have estimated the influence of temperature on the conformation of the ODS ligands by using the results of the calculation.

\section{Experimental}

\section{Molecular modeling}

The molecular model consisted of three parts; i.e. amorphous silica-base, dimethyloctadecylsilyl (ODS) ligands and solvent molecules of mobile phase. Ohta et al. studied the separation of fullerenes with $n$-hexane as the mobile phase, and reported that the molecular selectivity was significantly changed at subambient temperature. ${ }^{29-32}$ Recent study also showed an undesired intermolecular interaction between the mobile phase solvent and silica-base occurred when using the methanol/water mixtures as the mobile phase in the MD simulations. ${ }^{42}$ Therefore, $n$-hexane was used as the mobile phase solvent in this study. At the beginning of the calculation, the models for the stationary phase and mobile phase molecules were constructed individually. The silica-base consisted of a randomly cross-linked monolayer, and 16 ODS ligand molecules are immobilized onto this silica-base. Two ODSsilica groups were located at face-to-face positions, so that they sandwiched the mobile phase solvent to prevent the undesired interaction between the solvent and the silica-base as described previously. ${ }^{42}$ The total number of the ODS ligand molecules in the molecular model, therefore, is 32 . The ODS ligands are arrayed in approximately $8 \AA$ intervals, as was estimated by a calculation based on the ligand density and the surface area of a commercially available ODS column, Develosil ODS-5 (Nomura Chemicals, Seto, Japan).

Three-dimensional periodic boundary conditions with the unit-cell dimension of $36 \times 38 \times 80 \AA$ were applied to generate a mimic of the molecular model. The positions of the silicon atoms and the oxygen atoms in the ODS-silica are fixed during the MD simulation. The $n$-hexane molecules, as the mobile phase, are randomly positioned and the conformation of ODS chains has an all-trans conformation orientation at the initial state of the simulation. After the uniformity of the mobile phase was established, the bonded phase was combined with the mobile phase for each molecular model calculation. Mobile phase solvent molecules were located around the ODS ligand molecules at the initial state. The number of the mobile phase molecules in the molecular model was simply calculated as the function of specific gravity, molecular weight and Avogadro constant. The total numbers of mobile phase molecules and atoms in the molecular model are 257 and 8130, respectively. All molecular models were constructed by Chem 3D Pro (CambridgeSoft, MA, USA).

\section{Computational methods}

In this study, the TINKER modeling package developed by Ponder $^{43}$ was used for the MD simulation, and the MM3 Forcefield ${ }^{44}$ was introduced to approximate the potential energy of the simulated systems. To avoid the undesirable contacts arising from the initial modeling process, the energy of each system was first minimized by the steepest descents according
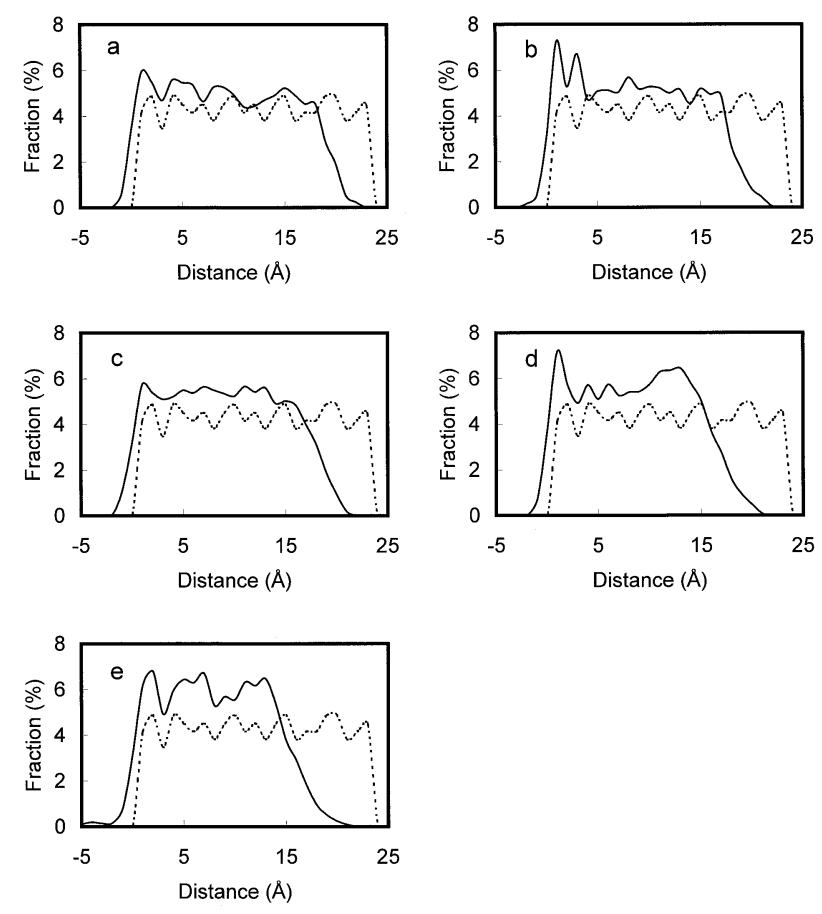

Fig. 1 Distribution fraction $v s$. the distances between carbon atoms of ODS ligands and the silica-gel surface. a, $210 \mathrm{~K}$; b, $240 \mathrm{~K}$; c, 270 $\mathrm{K}$; d, $300 \mathrm{~K}$; e, $330 \mathrm{~K}$; dashed-line, initial state.

to the limited memory Broyden-Fletcher-Goldfarb-Shanno (LBFGS) method until the deviation was decreased to $0.1 \mathrm{kcal}$ $\mathrm{mol}^{-1} \AA^{-1}{ }^{43}$ The systems were then subjected to a constantvolume MD simulation with the periodic boundary conditions. The Canonical Ensemble was chosen as the temperaturecontrolled algorithm, and the temperature of simulation was set at $210,240,270,300$ or $330 \mathrm{~K}$. The initial velocities were randomly assigned according to a Maxwell-Boltzmann distribution for the given temperature. The equations were solved by the Verlet algorithm with an integration time step of 1 fs. The length of the molecular-dynamics run was 100 ps (100000 time steps). The resulting atomic coordinates were stored on a disk after every 200 steps for the simulation analysis. Following the MD simulation, a number of physical parameters were derived to characterize the molecular properties of each simulated system. In this study, the information about the distribution of the ODS ligands and the solvent molecules in each simulation were used to interpret the results of the MD simulation.

\section{Results and Discussion}

The distribution of the ODS ligands and mobile phase molecules during MD simulation

Following the simulation, the last 100 of 500 the coordinate files that have been corrected by the MD simulation were used to characterize the surface structure of the bonded phase. The trajectory of potential energy obtained from MD simulations indicated that the molecular models are equilibrated enough when the number of time steps reached 5000 steps during each simulation run.

The distribution profiles of overall carbon atoms in ODS ligands $v s$. the distance from the silica-gel surface at each temperature condition are plotted in Fig. 1. The base-plane of 


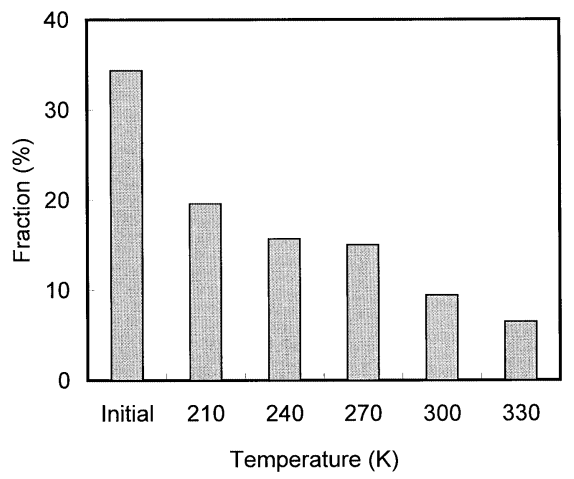

Fig. 2 Fraction of carbon atoms of ODS ligands located beyond 16 $\AA$ for each MD simulation.

the silica-gel surface was calculated from the coordinates of the silicon atoms in the dimethyloctadecylsilyl functional group. It was seen that the distribution ranges of the ODS ligands were narrowed compared with the range obtained from the initial state at all the simulated temperatures. At the initial state, all the alkyl chains were not bent and the carbon atoms were almost uniformly distributed in the range between $c a .1 \AA$ and $24 \AA$, while the distribution at the higher part (over $16 \AA$ ) was decreased at all temperatures during the MD simulations. The results indicate that the alkyl ligands were bent at all temperatures. Additionally, it is also suggested that an elevated temperature induced the more bended conformation of the ODS ligands.

Although the surface structures of the ODS-bonded phase during MD simulations were characterized by the distribution profiles of the carbon atom in the ODS ligand molecules, the influence of the simulation temperature on the surface structure was not completely illustrated. For the distribution profiles of the ODS ligands, the population of carbon atoms in the ODS ligands, which are located over $16 \AA$ for each molecular model, are illustrated in Fig. 2 to obtain further information on the relationship between the ligand conformation and the simulation temperatures. A significant reduction compared with the fraction at the initial model was clearly observed for all the simulated cases. While ca. $35 \%$ of all carbon atoms were distributed at the initial state, only up to $19.6 \%$ of the total carbon atoms were distributed at the distance over $16 \AA$ for all the simulated temperatures. Furthermore, it was more clearly seen compared to the results shown in Fig. 1, that if the simulation temperature was increased from 210 to $330 \mathrm{~K}$, the population of carbon atoms at the distance over $16 \AA$ was dramatically decreased from 19.6 to $6.5 \%$.

The distribution profiles of carbon atoms into $n$-hexane at each temperature are also plotted in Fig. 3. For the distribution of the $n$-hexane molecules, only a small difference of the distribution profiles was observed compared with the distribution of the ODS ligands at each temperature. However, the characteristic differences between the initial and simulated models were found in the region over $16 \AA$ for the distribution of $n$-hexane molecules. For the initial model, the distribution was relatively uniform over the range from $1 \AA$ to $25 \AA$, and the distribution fractions were significantly increased beyond $26 \AA$. In the case of low temperatures $(210-270 \mathrm{~K})$, however, the constant region was reduced and the distribution fraction was increased at $19 \AA$. This trend was more apparently observed in the cases of high temperatures $(300 \mathrm{~K}$ and $330 \mathrm{~K})$, where the distribution was increased at $17 \AA$. These constant areas of each
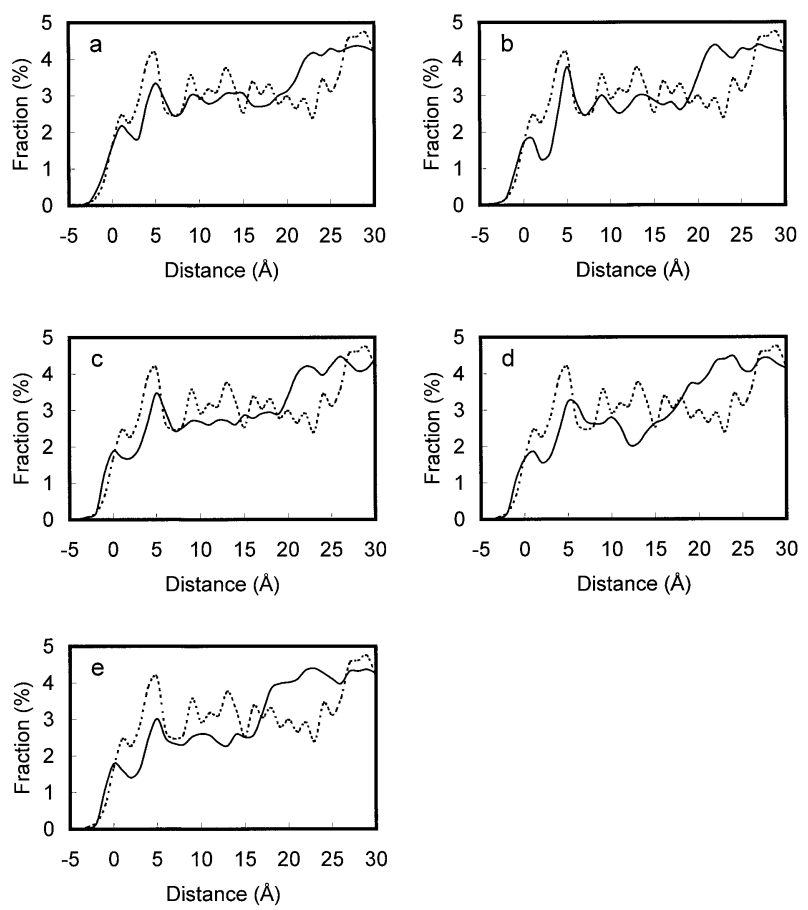

Fig. 3 Distribution fraction $v s$. the distance between carbon atoms of $n$-hexane and the silica-gel surface. a, $210 \mathrm{~K}$; b, $240 \mathrm{~K} ; \mathrm{c}, 270 \mathrm{~K}$; d, $300 \mathrm{~K}$; e, $330 \mathrm{~K}$; dashed-line, initial state.

distribution profile corresponded well to the distribution ranges obtained from the ODS ligands at each condition. The results suggest that the motion of the ODS ligands during the simulation depended on the temperature, and the numbers of the $n$-hexane molecules located around the ODS ligands were reduced from the initial state due to the mobility of the ODS ligands. It is also apparent that the $n$-hexane molecules were more movable than the ODS ligands in the molecular model during the simulations applied in this study. This is because the molecular sizes of $n$-hexane were relatively small, and they do not have any restrictions as like immobilized ODS ligands, and also not have relatively high intermolecular interaction as like hydrogen-bonds. Therefore, a part of the $n$-hexane molecules, which solvated with the ODS ligands, were moved away from the ODS ligands due to both phenomena: the high $n$-hexane mobility and the motion of ODS ligands.

\section{The mobility of molecules during the MD simulation}

The distribution profiles of carbon atoms only give us the information on the position of the carbon atoms in the molecular model during the simulation, and they cannot directly give the information about the mobility of the atoms. The comparison of the distribution profiles of the initial state and the simulated state are useful to estimate the mobility of molecules, however, further research is needed to obtain the detailed information on the molecular mobility during the simulations.

The time dependence of the average mean squared displacement (MSD) is one of the tools to obtain the information on the atom mobility. It is calculated according to the following relationship: ${ }^{45}$

$$
\operatorname{MSD}=\Sigma\left|r_{\mathrm{i}}\left(t+t_{0}\right)-r_{\mathrm{t}}\left(t_{0}\right)\right|^{2}
$$

Here, $\mathrm{i}$ is the number of carbon atoms and $r\left(t_{0}\right)$ is the initial coordination values. In this study, the coordinate files at $80 \mathrm{ps}$ 


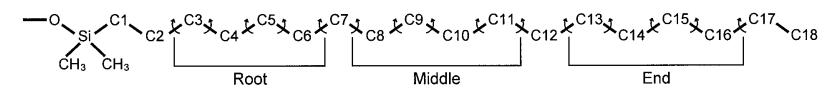

Fig. 4 Schematic structure of an $n$-octadecyldimethylsilyl ligand, in which are assigned carbon numbers and in which the torsion angles and classification of the three parts are indicated.
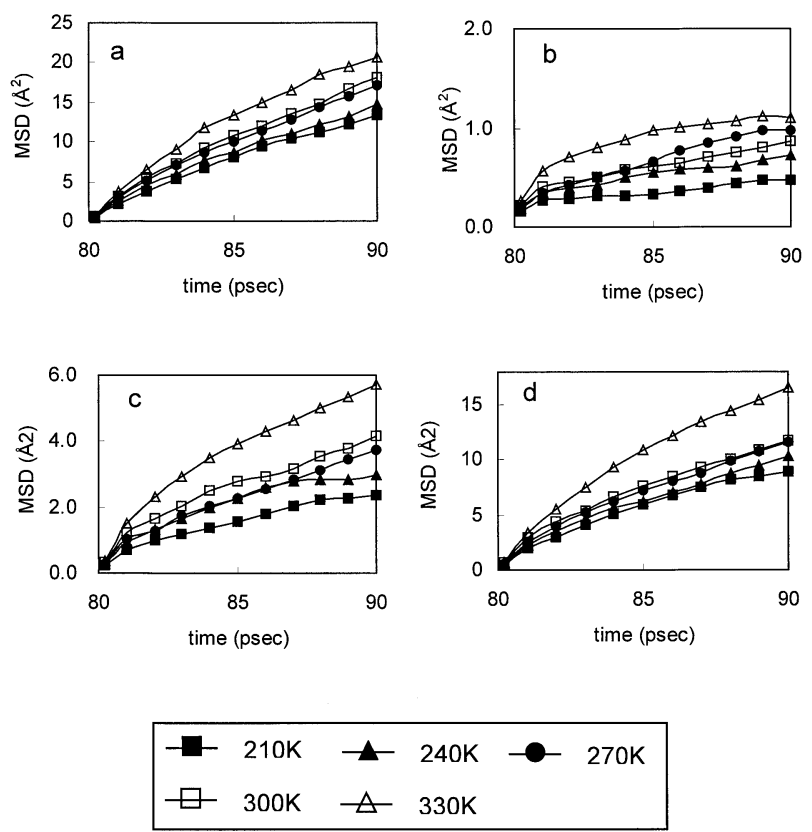

Fig. 5 Mean square displacements (MSDs) of carbon atoms in the $n$-hexane and the ODS ligand molecules. a, $n$-Hexane; b, C1*; c, C9*; d, C18*. *Assigned in Fig. 4.

during each MD simulation were used as the initial values.

To estimate the mobility of the carbon atoms individually, the carbon atoms in an ODS ligand were assigned in the order from $\mathrm{C} 1$ to $\mathrm{C} 18$, as illustrated in Fig. 4. Plots of the MSDs for the carbon of $n$-hexane molecules and $\mathrm{C} 1$ (nearest from the silicagel), C9 (middle positioned) and C18 (terminal) in the ODS ligands are shown in Fig. 5. Although the distribution profiles calculated from each temperature condition have small differences, the MSD plots indicated that increased temperature induces an increased motion of the mobile phase molecules (Fig. 5a). For the ODS ligands, it was clearly seen in Figs. 5b $d$ that the mobility of the carbon atoms of ODS ligands was increased when the carbon atoms were away from the silica-gel surface. It was also seen in Fig. 5 that the influence of temperature on the motion of carbon atoms was increased according to the increase of the distance between the silica-gel surface and the ODS ligands. For the plot of C18, the largest difference of the ligand mobility at each temperature was observed. It is especially interesting that a remarkable increase of the mobility was indicated at $330 \mathrm{~K}$. This temperature is so much higher than the temperature of the melting point of $n$ octadecane ( $c a .301-303 \mathrm{~K})$, that the ODS ligands show the liquid-like behavior. For the case of $300 \mathrm{~K}$, such a remarkable increase of the molecular mobility was not observed. This is because the temperature is close to the melting point of $n$ octadecane, and the time step of MD simulation was so short that the phase transition was not observed at this temperature.

The diffusion coefficient is another measure to estimate the relative mobilities of individual carbon atoms. It is known that

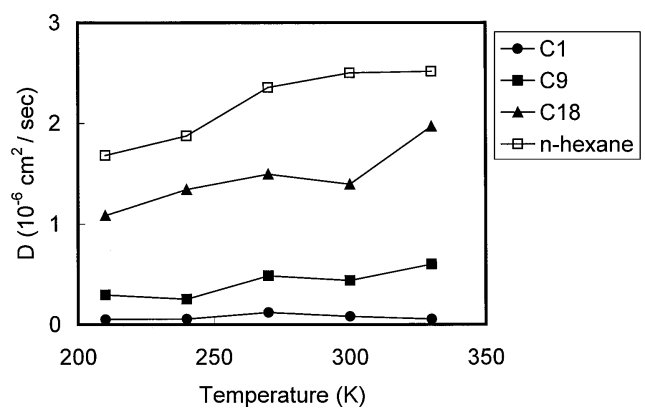

Fig. 6 Diffusion coefficients of carbon atoms in the $n$-hexane and the ODS ligand molecules. a, $n$-hexane; b, C1*; c, C9*; d, C18*. *Assigned in Fig. 4.

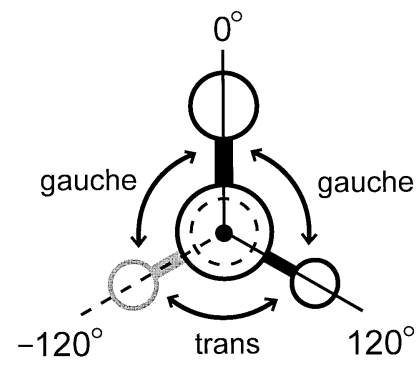

Fig. 7 Newman projection view of the part of the ODS ligand.

diffusion coefficients can be estimated from the slope of MSD plots using the Einstein relation as follows: ${ }^{45}$

$$
D=\frac{1}{6} \frac{\mathrm{d}}{\mathrm{d} t}\left\langle\left|r-r_{0}\right|^{2}\right\rangle
$$

The diffusion coefficients of each carbon atom were estimated by using the slopes from 84 to 90 ps of MSDs in Fig. 5, and these plots are illustrated in Fig. 6. It can be also seen from Fig. 6 that the diffusion coefficients increased in the order from $\mathrm{C} 1$ to $\mathrm{C} 18$ at each temperature. Interestingly, the motions of the terminal carbons (C18) were dramatically increased compared with the motions of $\mathrm{C} 1$ and $\mathrm{C} 9$. This trend corresponds well to the other data from the MD simulation, which was previously reported by Beck et al. ${ }^{33,34}$ and Hearn et al. ${ }^{36}$ In this study, the diffusion coefficient of $\mathrm{C} 18$ was ca. $2.0 \times 10^{-6} \mathrm{~cm}^{2} / \mathrm{s}$ at $330 \mathrm{~K}$; however, this value is an order of magnitude smaller than the previously reported data, which were observed from $\mathrm{C}_{8}$ phase ${ }^{33}$ and $\mathrm{C}_{18}$ phases. ${ }^{34,36}$ This is because the motions of ODS ligands were restricted by the $n$-hexane molecules solvated with the ODS ligands.

\section{Trans-gauche statistics}

The population of the gauche fraction in the ODS ligand conformation is also a good index to estimate the surface structure of the bonded phase. We have calculated the dihedral angle of the overall ODS ligand molecules, and those are defined in three parts as shown in Fig. 4. The trans and gauche conformations of each torsional axis are classified by the dihedral angles, as illustrated in Fig. 7.

In Fig. 8, the fraction of gauche conformation of root, middle and end part of the ODS ligands at each temperature is illustrated. For all parts, it can be seen that the gauche fraction of the ODS ligands will be higher for all models when the temperature is increased. This trend is the same as the experimentally observed trends by NMR, ${ }^{5,7}$ FTIR, ${ }^{5,8}$ and Raman 


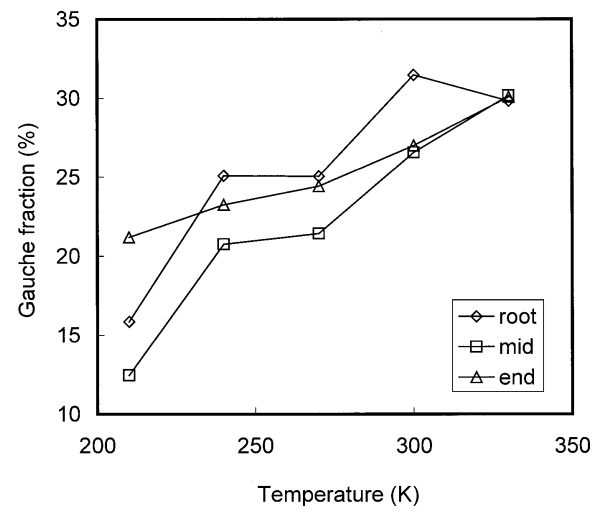

Fig. 8 Fraction of gauche dihedral angles (gauche fraction) in ODS ligands at each temperature.

spectroscopies. ${ }^{11-13} \quad$ Some detailed trends were seen by comparing the populations at each part. It is interesting that the gauche population for the end part was linearly increased according to the increase of the temperature, while the other population for the parts were not linearly increased. The ODS ligand conformation is mainly decided by the intermolecular interactions of adjacent ODS ligands and those among the ODS lingands and $n$-hexane molecules. The balance of these interactions can explain the difference of the gauche populations at each part. The former interaction plays a role to restrict the motion of ODS ligands and its conformation is more ordered when this interaction is dominant. The latter interaction activates the molecular motion of the ODS ligands, and a more bent conformation is seen when this interaction is dominant. At the end part, a relatively large number of $n$-hexane molecules interacted with the ODS ligands, as seen in the distribution profiles in Figs. 1 and 3; then the intermolecular interaction between $n$-hexane and ODS ligands were dominant. For the cases of the root and middle parts, the intermolecular interaction of adjacent ODS ligands is dominant because the mobile phase solvent molecules were moved out by their high mobility. Therefore, the motion of ODS ligands was more restricted, resulting a more fixed conformation. The higher gauche populations observed at the root part can be explained if the bending process occurred due to the elimination of $n$-hexane molecules at the early stage in the MD calculation. This suggestion also well explains the relatively low gauche population observed at $210 \mathrm{~K}$. This is because the molecular mobility of $n$-hexane was quite low at this temperature and thus the motion of ODS ligands was restricted by the intermolecular interaction between the ODS ligands and $n$-hexane molecules.

\section{Acknowledgements}

The authors thank Prof. J. W. Ponder, Biochemistry \& Molecular Biophysics, Washington University School of Medicine for developing the freely available TINKER software package.

\section{References}

1. D. W. Sindorf and G. E. Maciel, J. Am. Chem. Soc., 1983 , 105, 1848.

2. E. Bayer, A. Paulus, B. Peters, G. Laupp, J. Reiners, and K.
Albert, J. Chromatogr., 1986, 364, 25.

3. E. C. Kelusky and C. A. Fyfe, J. Am. Chem. Soc., 1986 , 108, 1746.

4. P. Shah, L. B. Rogers, and J. C. Fetzer, J. Chromatogr., 1987, 388, 411.

5. K. Jinno, T. Ibuki, N. Tanaka, M. Okamoto, J. C. Fetzer, W. R. Biggs, P. R. Griffiths, and J. M. Olinger, J. Chromatogr., 1989, 461, 209.

6. M. E. Gangoda and R. K. Gilpin, Langmuir, 1990, 6, 941.

7. H. Ohta, Y. Saito, K. Jinno, J. J. Pesek, M. T. Matyska, Y.-L. Chen, J. Archer, J. C. Fetzer, and W. R. Biggs, Chromatographia, 1995, 40, 507.

8. M. Pursch, L. C. Sander, and K. Albert, Anal. Chem., 1996, 68, 4107.

9. L. C. Sander, J. B. Callis, and L. R. Field, Anal. Chem., 1983, 55, 1068.

10. W. R. Thompson and J. E. Pemberton, Anal. Chem., 1994, 66,3362 .

11. M. Ho, M. Cai, and J. E. Pemberton, Anal. Chem., 1997, 69, 2613.

12. M. Ho and J. E. Pemberton, Anal. Chem., 1998, 70, 4915.

13. J. E. Pemberton, M. Ho, C. J. Orendorff, and M. W. Ducey, J. Chromatogr. A, 2001, 913, 243.

14. M. W. Ducey, Jr., C. J. Orendorff, J. E. Pemberton, and L. C. Sander, Anal. Chem., 2002, 74, 5576.

15. M. W. Ducey, Jr., C. J. Orendorff, J. E. Pemberton, and L. C. Sander, Anal. Chem., 2002, 74, 5585.

16. C. J. Orendorff, M. W. Ducey, Jr., J. E. Pemberton, and L. C. Sander, Anal. Chem., 2003, 75, 3028.

17. C. J. Orendorff, M. W. Ducey, Jr., J. E. Pemberton, and L. C. Sander, Anal. Chem., 2003, 75, 3037.

18. M. E. Montgomery, Jr., M. A. Green, and M. J. Wirth, Anal. Chem., 1992, 64, 1170.

19. C. A. Fung, K. Fung, and M. F. Burke, J. Chromatogr. A, 1996, $752,41$.

20. K. Jinno, T. Nagoshi, N. Tanaka, M. Okamoto, J. C. Fetzer, and W. R. Biggs, J. Chromatogr., 1988, 436, 1.

21. L. C. Sander and S. A. Wise, Anal. Chem., 1984, 56, 504.

22. L. C. Sander and S. A. Wise, Anal. Chem., 1987, 59, 2309.

23. K. Jinno, S. Shimura, N. Tanaka, K. Kimata, J. C. Fetzer, and W. R. Biggs, Chromatographia, 1989, 27, 285.

24. K. B. Sentell and J. G. Dorsey, J. Chromatogr., 1989, 461, 193.

25. L. C. Sander and S. A. Wise, Anal. Chem., 1995, 67, 3284.

26. C. A. Rimmer, L. C. Sander, S. A. Wise, and J. G. Dorsey, J. Chromatogr. A, 2003, 1007, 11.

27. L. C. Sander and S. A. Wise, Anal. Chem., 1989, 61, 1749.

28. C. M. Bell, L. C. Sander, and S. A. Wise, J. Chromatogr. A, 1997, 757, 29.

29. H. Ohta, Y. Saito, K. Jinno, H. Nagashima, and K. Itoh, Chromatographia, 1994, 39, 453.

30. H. Ohta, K. Jinno, Y. Saito, J. C. Fetzer, W. R. Biggs, J. J. Pesek, M. T. Matyska, and Y.-L. Chen, Chromatographia, 1996, 42, 56 .

31. H. Ohta, Y. Saito, N. Nagae, J. J. Pesek, M. T. Matyska, and K. Jinno, J. Chromatogr. A, 2000, 883, 55.

32. Y. Saito, H. Ohta, and K. Jinno, Anal. Chem., 2004, 76, 266A.

33. S. J. Klatte and T. L. Beck, J. Phys. Chem., 1993, 97, 5727.

34. S. J. Klatte and T. L. Beck, J. Phys. Chem., 1995, 99, 16024.

35. S. J. Klatte and T. L. Beck, J. Phys. Chem., 1996, 100, 5931.

36. I. Yarovsky, M.-I. Aguilar, and M. T. W. Hearn, Anal. Chem., 1995, 67, 2145. 
37. M. R. Schure, "Chemically Modified Surfaces", ed. J. J. Pesek and I. E. Leigh, 1994, Royal Society of Chemistry, Cambridge, England, 181.

38. M. R. Schure, "Advances in Chromatography Series", ed. P. R. Brown and E. Grushka, 1998, Vol. 39, Marcel Dekker, NewYork, USA, 139.

39. J. T. Slusher and R. D. Mountain, J. Phys. Chem. B, 1999, 103, 1354.

40. K. Ban and K. Jinno, Anal. Sci., 2001, 17, 113.

41. C.-Y. Cheng, T.-L. Chen, and B.-C. Wang, J. Mol. Struct.
(Theochem), 2002, 577, 81.

42. K. Ban, Y. Saito, and K. Jinno, Anal. Sci., 2004, 20, 1403.

43. J. W. Ponder, "TINKER: Software Tools for Molecular Design, Ver. 4.1", 2003, Washington University School of Medicine..

44. N. L. Allinger, Y. H. Yuh, and J.-H. Lii, J. Am. Chem. Soc., 1989, $111,8551$.

45. A. Ueda, "Computer Simulation", 1990, Asakura Shoten, Tokyo, Japan. 